\title{
OBSERVATIONS ON THE EFFECT OF STREPTOCOCCAL UPPER RESPIRATORY INFECTIONS ON RHEUMATIC CHILDREN : A THREE-YEAR STUDY ${ }^{1}$
}

\author{
BY ANN G. KUTTNER AND ELMA KRUMWIEDE \\ (From Irvington House, Irvington-on-Hudson, N. Y.)
}

(Received for publication December 3, 1940)

The relationship of beta hemolytic streptococci to the etiology of rheumatic fever has long been a controversial subject. Although many students (1) of this disease are of the opinion that both the initial attack and subsequent recurrences of rheumatic fever are usually preceded by streptococcal upper respiratory infections, others (2) believe that this association is merely accidental. At the present time, there is no adequate explanation of the exact rôle played by streptococci in the etiology of rheumatic fever. On the other hand, the association between streptococcal upper respiratory infections and rheumatic fever seems too constant to be completely disregarded.

It is obvious that many factors which cannot be adequately studied in a general hospital or in ambulatory rheumatic patients enter into this problem. Studies of this kind can be carried out more satisfactorily in sanatoria where the patients are relatively isolated and are under careful medical supervision for long periods of time. Since the severity of both streptococcal upper respiratory infections and rheumatic fever is known to vary from year to year, it was thought that a comparison of the results obtained during three successive winters would yield more reliable information than observations limited to a single year.

\section{PLAN OF STUDY}

The same methods and procedures were used during three successive years, 1937 to 1940.

\section{Type of institution}

Irvington House, a sanatorium for rheumatic children, is situated in the country, 20 miles from New York City. The children were admitted from the cardiac clinics of various hospitals in Greater New York. Children who had had one or more attacks of polyarthritis or carditis without marked cardiac damage were selected. With a few exceptions, children with histories of "pure" chorea without polyarthritis or carditis were excluded. New

1 This work was aided by a grant from The Commonwealth Fund. cases were admitted only during the summer and fall months. Each year 108 suitable patients, consisting of new children and children remaining longer than one winter, were assembled by the first of December. This group then remained in the institution until the end of May or longer.

The children lived and went to school in the same building. They had no contact with other children and each child was permitted only two adult visitors every six weeks. The group consisted of 66 girls and 42 boys varying in age from 6 to 15 years.

\section{Routine procedures}

Rectal temperatures and pulse rates were taken three times daily. Leukocyte counts, hemoglobin estimations, and erythrocyte sedimentation rates were done routinely every three or four weeks, or more often when necessary. Children who had symptoms of upper respiratory infections were put to bed and isolated in cubicles, or when possible, in separate rooms. Leukocyte counts were taken on the first or second day of illness.

\section{Bacteriological procedures}

Throat cultures to determine the presence of Group A beta hemolytic streptococci were taken routinely once a week on every child throughout the year. Additional cultures were taken on two successive days on children who developed symptoms of any kind. Swabs were streaked on freshly prepared 5 per cent sheep blood agar plates (3). After eighteen-hour growth, hemolytic colonies were re-streaked and fished to broth. Attempts were made to type all Group A beta hemolytic streptococci isolated. Both the slide agglutination method of Griffith (4) and the precipitin reaction of Lancefield (5) with “ $M$ " extracts were used. In most instances, cultures were considered to be successfully typed only when the findings obtained by slide agglutination could be confirmed by the precipitin reaction. Many strains were isolated which could not be typed by either method. In several instances, agglutinating and precipitating sera were prepared by immunizing rabbits with these cultures.

Antistreptolysin $\mathrm{O}$ titers (6) were determined every three to six weeks, and more often following upper respiratory infections or during rheumatic activity. Antistreptolysin S titers (6) were not determined.

\section{Definitions}

It has been noted by several observers that the clinical symptoms of the streptococcal upper respiratory infec- 
tions preceding the development of rheumatic recurrences may be extremely mild and therefore can be easily overlooked.

The cases of streptococcal upper respiratory infections reported in this study were in most instances clear-cut. The children complained of sore throat and the pharynx usually appeared inflamed. Occasionally exudate was present. Most of the patients had fever and elevated white blood counts. Throat cultures taken on the first or second day of illness showed varying numbers of Group A beta hemolytic streptococci of a type which had not been present previously.

In certain instances, however, especially during the winter of 1937 to 1938 , it was difficult to decide whether a child really had an upper respiratory infection or had merely become a streptococcus carrier. A few children who had neither complaints nor symptoms, but whose white blood counts showed a definite rise coincident with the appearance of streptococci in their throat culture, or whose antistreptolysin $O$ titer rose three to six weeks later, were considered to be cases of upper respiratory infection.

Children in whom the appearance of streptococci in their routine throat cultures was not accompanied by symptoms, or by a rise in their white blood counts or in their antistreptolysin $\mathrm{O}$ titer, were considered to have become carriers, either temporary or chronic.

It is well known that the course of rheumatic fever varies greatly. In some children, evidence of low-grade activity persists for months and years. This type of case is generally described as continuous or polycyclic. Slight exacerbations of the symptoms may occur from time to time in these children without apparent precipitating cause. Other children are subject to severe, acute attacks but between attacks clinical or laboratory signs of rheumatic activity are not demonstrable. It is in this monocyclic type of case that factors tending to precipitate rheumatic recurrences can be studied most satisfactorily.

\section{Streptococcal upper respiratory infections occurring durng the winter of 1937 to 1938}

During this winter, 12 of the 108 children developed upper respiratory infections associated with Group A beta hemolytic streptococci of a single type. The organisms isolated from these cases did not belong to any of the known serological types of streptococci. Agglutinating and precipitating sera were therefore prepared by immunizing rabbits with a culture, C51, isolated from one of these patients (Case 2, Table I). By means of slide agglutination and precipitin tests, the organisms isolated from the other 11 patients were shown to belong to the same serological type as C51.2

The symptoms produced by this infection were mild. The 12 cases appeared sporadically over a period of six months, the first occurring in December 1937 and the last in May 1938. Prior to this infection, 2 of the 12 children had continuously elevated erythrocyte sedimentation rates, and the rheumatic infection in these 2 individuals therefore was not considered to be quiescent. Neither of these 2 children had exacerbations of their symptoms following the upper respiratory infection. The remaining 10 children had not had signs of rheumatic activity for six months or longer. Of these 10 children, 6 developed rheumatic recurrences after latent periods varying from nine to eighteen days. Five of the 6 children who developed rheumatic recurrences showed a prompt rise in antistreptolysin $\mathrm{O}$ titer. Three children who did not develop recurrences also showed a definite rise in titer (Cases 5, 6 and 9). The data in regard to these 12 cases are presented in Table $\mathrm{I}$.

Ten children became carriers of streptococcus C51. None of them had rheumatic recurrences. Except for 3 children who showed continuous low-grade activity, none of the other 83 children in the institution who did not contract infections with streptococcus C51 developed symptoms of rheumatic activity.

\section{Streptococcal upper respiratory infections occurring during the winter of 1938 to 1939}

None of the children developed streptococcal upper respiratory infections during December and January. Beginning in February 1939 and continuing through June, 32 children developed upper respiratory infections associated with Group A beta hemolytic streptococci of a single type, Type 4. The majority of these infections were definitely more severe than those associated with streptococcus C51 which had occurred during the previous winter. Many of the children had fever of 101 to $103^{\circ} \mathrm{F}$. for two or three days. The

2 Nine of these cultures and some agglutinating serum were sent to Dr. Fred Griffith in England. He agreed that these strains did not belong to any of the known Group A streptococcal types and that they were serologically identical. 
TABLE I

Data on streptococcal upper respiratory infections with streptococcus C51 and rheumatic sequelae during the winter 1937 to 1938

\begin{tabular}{|c|c|c|c|c|c|c|c|c|c|c|c|c|}
\hline \multirow{2}{*}{$\begin{array}{c}\text { Case } \\
\text { num- }\end{array}$} & \multicolumn{3}{|c|}{ Patient } & \multirow{2}{*}{$\begin{array}{l}\text { Previous } \\
\text { rheumatic } \\
\text { attacks } \\
\text { and age } \\
\text { at each }\end{array}$} & \multirow[b]{2}{*}{ Date } & \multirow[b]{2}{*}{ Clinical findings } & \multirow{2}{*}{$\begin{array}{c}\text { Strep- } \\
\text { tococ- } \\
\text { cus } \\
\text { C51. } \\
\text { num- } \\
\text { ber of } \\
\text { colonies }\end{array}$} & \multirow{2}{*}{$\begin{array}{c}\text { La- } \\
\text { tent } \\
\text { period }\end{array}$} & \multicolumn{4}{|c|}{ Blood } \\
\hline & Name & Age & Sex & & & & & & Date & $\begin{array}{l}\text { White } \\
\text { blood } \\
\text { cells }\end{array}$ & $\begin{array}{l}\text { Sedi- } \\
\text { ment- } \\
\text { ation } \\
\text { rate* }\end{array}$ & $\begin{array}{l}\text { Anti- } \\
\text { strepto- } \\
\text { lysin 0 } \\
\text { titer }\end{array}$ \\
\hline 1 & $\underset{\text { J264 }}{\text { J.R. }}$ & $\begin{array}{c}\text { years } \\
8\end{array}$ & $\mathbf{F}$ & $\begin{array}{l}6 \mathrm{Ch} \dagger \\
7 \mathrm{Ch}\end{array}$ & $\begin{array}{l}\text { December 27, } 1937 \\
\text { January } 12,1938\end{array}$ & $\begin{array}{l}\text { Pharyngitis. No fever. } \\
\text { Mild carditis. }\end{array}$ & few & $\begin{array}{c}\text { days } \\
16\end{array}$ & $\begin{array}{lr}\text { November } 15 \\
\text { December } & 10 \\
& \\
\text { December } & 27 \\
\text { January } & 13 \\
\text { January } & 19 \\
\text { January } & 24 \\
\text { January } & 30 \\
\text { February } & 4 \\
\text { February } & 20\end{array}$ & $\begin{array}{l}5,000 \\
\\
8,800 \\
8,800 \\
7,100 \\
6,500\end{array}$ & $\begin{array}{r}\mathbf{m m} . \\
2 \\
5 \\
31 \\
31 \\
30 \\
21 \\
4 \\
6\end{array}$ & $\begin{array}{r}\text { units } \\
100 \\
80 \\
100 \\
100 \\
100 \\
100\end{array}$ \\
\hline 2 & $\underset{3236}{\text { A.C. }}$ & 11 & $\mathbf{M}$ & $\begin{array}{l}3 \mathrm{P} \text { and } \mathrm{C} \\
5 \mathrm{P} \text { and } \mathrm{C} \\
6 \mathrm{C} \\
8 \mathrm{P} \text { and } \mathrm{C} \\
9 \mathrm{P} \text { and } \mathrm{C}\end{array}$ & $\begin{array}{l}\text { January 3, } 1938 \\
\text { January } 15,1938\end{array}$ & $\begin{array}{l}\text { None. } \\
\text { Severe carditis, second de- } \\
\text { gree heart block, nodules, } \\
\text { erythema. }\end{array}$ & 50 & 12 & \begin{tabular}{|rr} 
December & 27 \\
December & 30 \\
& \\
January & 10 \\
January & 13 \\
& \\
January & 16 \\
January & 19 \\
January & 23 \\
January & 30 \\
February & 12 \\
March & 5
\end{tabular} & $\begin{array}{r}7,800 \\
11,500 \\
\\
22,700 \\
24,500 \\
15,200 \\
9,900\end{array}$ & $\begin{array}{r}1 \\
6 \\
6 \\
24 \\
30 \\
30 \\
31 \\
30\end{array}$ & 80 \\
\hline 3 & $\begin{array}{l}\text { C.F. } \\
3269\end{array}$ & 10 & $\mathbf{M}$ & $\begin{array}{l}7 \mathrm{Ch} \\
7 \mathrm{P} \\
7 \mathrm{P} \text { and } \mathrm{C} \\
8 \mathrm{Ch}\end{array}$ & January 7, 1938 & $\begin{array}{l}\text { Pharyngitis. Fever } 100 \text { to } \\
102.6^{\circ} \text { for one day. } \\
\text { No rheumatic sequelae. }\end{array}$ & 15 & & $\begin{array}{lr}\text { December } & 21 \\
& \\
\text { January } & 8 \\
\text { January } & 10 \\
\text { January } & 21 \\
\text { January } & 24 \\
\text { February } & 5 \\
\text { February } & 7 \\
\text { February } & 11 \\
\end{array}$ & $\begin{array}{r}7,600 \\
12,400 \\
7,800 \\
5,400 \\
7,800\end{array}$ & $\begin{array}{r}10 \\
6 \\
9 \\
21 \\
7\end{array}$ & $\begin{array}{r}80 \\
100 \\
100 \\
100 \\
100\end{array}$ \\
\hline 4 & $\begin{array}{l}\text { W.C. } \\
3294\end{array}$ & 11 & $\mathbf{M}$ & $7 \mathbf{P}$ & January 27, 1938 & $\begin{array}{l}\text { Pharyngitis. Fever } 100 \text { to } \\
101.4^{\circ} \text { for two days. } \\
\text { No rheumatic sequelae. }\end{array}$ & $75 \%$ & & $\begin{array}{lr}\text { January } & 18 \\
\text { January } & 23 \\
& \\
\text { January } & 27 \\
\text { January } & 28 \\
\text { February } & 4 \\
\text { February } & 11 \\
\text { February } & 20 \\
\text { March } & 1 \\
\text { March } & 14 \\
\end{array}$ & $\begin{array}{l}4,400 \\
7,600 \\
4,850 \\
4,800 \\
5,200\end{array}$ & $\begin{array}{r}6 \\
28 \\
28 \\
22 \\
17 \\
13 \\
13 \\
6\end{array}$ & $\begin{array}{l}50 \\
50 \\
50 \\
50 \\
50 \\
50 \\
60\end{array}$ \\
\hline 5 & $\begin{array}{l}\text { G.H. } \\
3278\end{array}$ & 13 & $\mathbf{F}$ & $9 \mathrm{P}$ and $\mathrm{C}$ & February 19, 1938 & $\begin{array}{l}\text { Pharyngitis. Fever } 100.4 \text { to } \\
102.6^{\circ} \text { for two days. } \\
\text { No rheumatic sequelae. }\end{array}$ & $75 \%$ & & $\begin{array}{lr}\text { February } & 13 \\
\text { February } & 16 \\
& \\
\text { February } & 19 \\
\text { February } & 21 \\
\text { February } & 22 \\
\text { February } & 26 \\
\text { March } & 12 \\
\text { March } & 21 \\
\text { April } & 7 \\
\text { April } & 14 \\
\text { April } & 20 \\
\text { April } & 25 \\
\end{array}$ & $\begin{array}{r}9,300 \\
11,600 \\
6,000 \\
6,400 \\
9,500 \\
8,300\end{array}$ & $\begin{array}{r}20 \\
10 \\
10 \\
9 \\
2 \\
6\end{array}$ & $\begin{array}{r}50 \\
50 \\
\\
100 \\
100 \\
250 \\
200 \\
100 \\
\end{array}$ \\
\hline 6 & $\underset{3283}{\text { H.S.t }}$ & 13 & $\mathbf{F}$ & $\begin{array}{r}5 \mathrm{Ch} \\
7 \mathrm{Ch} \\
10 \mathrm{Ch} \\
11 \mathrm{Ch}\end{array}$ & February 22, 1938 & $\begin{array}{l}\text { Pharyngitis. Fever } 100.4^{\circ} \\
\text { for one day. } \\
\text { Mild sinusitis after twenty- } \\
\text { eight days, left antrum } \\
\text { washed. } \\
\text { No rheumatic sequelae. }\end{array}$ & $25 \%$ & & $\begin{array}{lr}\text { January } & 22 \\
\text { February } & 12 \\
\text { February } & 16 \\
& \\
\text { February } & 22 \\
\text { February } & 26 \\
\text { February } & 28 \\
\text { March } & 5 \\
\text { March } & 12 \\
\text { March } & 22 \\
\text { March } & 28 \\
\text { March } & 30 \\
\text { April } & 7 \\
\text { April } & 11 \\
\text { April } & 14\end{array}$ & $\begin{array}{r}8,200 \\
14,800 \\
9,900 \\
12,800 \\
8,000 \\
7,500\end{array}$ & $\begin{array}{l}18 \\
22 \\
21 \\
34 \\
32 \\
28 \\
29\end{array}$ & $\begin{array}{c}60 \\
100 \\
100 \\
200 \\
165\end{array}$ \\
\hline
\end{tabular}

* Erythrocyte sedimentation rate, Wintrobe-Landsberg method, values over $10 \mathrm{~mm}$. are abnormal.

$\uparrow$ The following abbreviations are used: $\mathrm{Ch}$ Chorea

P Polyarthritis

C Carditis

Jt $P$ Joint pains

H D Heart disease diagnosed.

† This patient had continuous low grade activity (elevated sedimentation rate), no exacerbation was observed. 
TABLE I (continued)

\begin{tabular}{|c|c|c|c|c|c|c|c|c|c|c|c|c|c|}
\hline \multirow[b]{2}{*}{$\begin{array}{c}\text { Case } \\
\text { num- } \\
\text { ber }\end{array}$} & \multicolumn{3}{|c|}{ Patient } & \multirow{2}{*}{$\begin{array}{l}\text { Previous } \\
\text { rheumatic } \\
\text { attacks } \\
\text { and age } \\
\text { at each }\end{array}$} & \multirow[b]{2}{*}{ Date } & \multirow[b]{2}{*}{ Clinical findings } & \multirow{2}{*}{$\begin{array}{c}\text { Strep- } \\
\text { tococ- } \\
\text { cus } \\
\text { C51. } \\
\text { num- } \\
\text { ber of } \\
\text { colonies }\end{array}$} & \multirow[b]{2}{*}{$\mid \begin{array}{c}\text { La- } \\
\text { tent } \\
\text { period }\end{array}$} & \multicolumn{5}{|c|}{ Blood } \\
\hline & Name & Age & Sex & & & & & & Date & & $\begin{array}{l}\text { White } \\
\text { blood } \\
\text { cells }\end{array}$ & $\begin{array}{l}\text { Sedi- } \\
\text { ment- } \\
\text { ation } \\
\text { rate* }\end{array}$ & $\begin{array}{l}\text { Anti- } \\
\text { strepto- } \\
\text { lysin 0 } \\
\text { titer } \\
\end{array}$ \\
\hline 7 & $\begin{array}{l}\text { M.D. } \\
3376\end{array}$ & $\begin{array}{c}\text { years } \\
14\end{array}$ & F & $\begin{array}{rl}8 & \mathrm{P} \\
9 \mathrm{P} \\
10 & \mathrm{P}\end{array}$ & $\begin{array}{l}\text { February 23, } 1938 \\
\text { March 9, } 1938\end{array}$ & $\begin{array}{l}\text { Pharyngitis. Fever } 100.6^{\circ} \\
\text { for one day. } \\
\text { Polyarthritis. }\end{array}$ & 12 & $\begin{array}{l}\text { days } \\
15\end{array}$ & $\begin{array}{l}\text { February } \\
\text { February } \\
\text { February } \\
\text { February } \\
\text { March } \\
\text { March } \\
\text { March } \\
\text { March } \\
\text { March } \\
\text { March } \\
\text { April }\end{array}$ & $\begin{array}{r}13 \\
25 \\
26 \\
28 \\
6 \\
9 \\
12 \\
16 \\
22 \\
28 \\
16\end{array}$ & $\begin{array}{r}9,200 \\
13,100 \\
8,700 \\
\\
16,600 \\
17,200 \\
11,500 \\
13,300 \\
9,600\end{array}$ & $\begin{array}{c}m m . \\
5 \\
15 \\
18 \\
\\
27 \\
19 \\
29 \\
12\end{array}$ & $\begin{array}{r}\text { urits } \\
80 \\
\\
80 \\
100 \\
\\
\\
250 \\
333 \\
333 \\
250\end{array}$ \\
\hline 8 & $\underset{3307}{\text { E.Mc. } \downarrow}$ & 11 & $\mathbf{F}$ & $\begin{array}{l}6 \mathrm{Ch} \\
7 \mathrm{Jt} P\end{array}$ & March 10, 1938 & $\begin{array}{l}\text { Pharyngitis. Fever } 100.4 \text { to } \\
101^{\circ} \text { for two days. } \\
\text { No rheumatic sequelae. }\end{array}$ & few & & $\begin{array}{l}\text { February } \\
\text { February } \\
\text { March } \\
\text { March } \\
\text { March } \\
\text { March } \\
\text { April } \\
\text { April } \\
\text { April } \\
\text { May } \\
\text { May }\end{array}$ & $\begin{array}{r}12 \\
25 \\
5 \\
12 \\
21 \\
28 \\
3 \\
17 \\
30 \\
6 \\
13\end{array}$ & $\begin{array}{l}6,100 \\
8,500 \\
5,500 \\
5,500 \\
5,000\end{array}$ & $\begin{array}{l}16 \\
21 \\
27 \\
29 \\
21 \\
29 \\
25 \\
21 \\
15 \\
13\end{array}$ & $\begin{array}{l}100 \\
100 \\
\\
100 \\
165 \\
165\end{array}$ \\
\hline 9 & $\begin{array}{l}\text { R.W. } \\
\text { 3381 }\end{array}$ & 11 & $\mathbf{F}$ & $9 \mathrm{P}$ and $\mathrm{C}$ & March 19, 1938 & $\begin{array}{l}\text { Pharyngitis. Fever } 100.2 \text { to } \\
102.4^{\circ} \text { for three days. } \\
\text { No rheumatic sequelae. }\end{array}$ & $50 \%$ & & $\begin{array}{l}\text { February } \\
\text { March } \\
\text { March } \\
\text { March } \\
\text { March } \\
\text { March } \\
\text { April } \\
\text { April } \\
\text { April } \\
\end{array}$ & $\begin{array}{l}19 \\
17 \\
19 \\
21 \\
22 \\
28 \\
10 \\
23 \\
30\end{array}$ & $\begin{array}{r}5,900 \\
19,800 \\
8,100 \\
8,100 \\
7,800 \\
\end{array}$ & $\begin{array}{r}16 \\
7 \\
1 \\
1 \\
1\end{array}$ & $\begin{array}{r}60 \\
80 \\
100 \\
80 \\
80\end{array}$ \\
\hline 10 & $\underset{3382}{\text { F.B. }}$ & 12 & $\mathbf{F}$ & $7 \mathrm{C}$ & $\begin{array}{l}\text { March 28, } 1938 \\
\text { April 12, } 1938\end{array}$ & $\begin{array}{l}\text { Pharyngitis. Fever } 100 \text { to } \\
102^{\circ} \text { for one day. } \\
\text { Polyarthritis, mild carditis } \\
\text { (PR interval prolonged). }\end{array}$ & 25 & 15 & $\begin{array}{l}\text { March } \\
\text { March } \\
\text { March } \\
\text { April } \\
\text { April } \\
\text { April } \\
\text { April } \\
\text { April } \\
\text { April } \\
\text { April } \\
\text { May } \\
\text { May } \\
\text { May }\end{array}$ & $\begin{array}{r}14 \\
21 \\
28 \\
3 \\
6 \\
10 \\
12 \\
16 \\
21 \\
30 \\
6 \\
16 \\
19\end{array}$ & \begin{tabular}{|r|}
6,700 \\
17,400 \\
10,600 \\
9,900 \\
16,500 \\
11,800 \\
11,600 \\
12,600 \\
8,200 \\
\end{tabular} & $\begin{array}{l}12 \\
17 \\
18 \\
18 \\
\\
31 \\
20 \\
30 \\
34 \\
25\end{array}$ & $\begin{array}{l}100 \\
100 \\
100 \\
\\
100 \\
250 \\
250 \\
250 \\
250\end{array}$ \\
\hline 11 & $\underset{3361}{\text { F.C. }}$ & 13 & $\mathbf{F}$ & $\begin{array}{rl}6 & \mathrm{P} \\
7 \mathrm{P} \\
10\end{array}$ & $\begin{array}{l}\text { April 5, } 1938 \\
\text { April 23, } 1938\end{array}$ & \begin{tabular}{|l|} 
Pharyngitis. No fever. \\
Carditis, mild (PR interval \\
prolonged).
\end{tabular} & 40 & 18 & $\begin{array}{l}\text { March } \\
\text { March } \\
\text { April } \\
\text { April } \\
\text { April } \\
\text { April } \\
\text { April } \\
\text { April } \\
\text { April } \\
\text { April } \\
\text { April } \\
\text { April } \\
\text { May } \\
\text { May } \\
\text { May } \\
\text { May } \\
\text { June }\end{array}$ & \begin{tabular}{r|}
4 \\
12 \\
2 \\
5 \\
7 \\
10 \\
17 \\
20 \\
22 \\
23 \\
25 \\
30 \\
4 \\
6 \\
12 \\
19 \\
9
\end{tabular} & \begin{tabular}{|r|}
6,900 \\
\\
8,000 \\
7,000 \\
7,500 \\
10,000 \\
10,000 \\
\\
8,100 \\
6,700 \\
9,400 \\
9,900 \\
4,700 \\
\end{tabular} & $\begin{array}{l}10 \\
12 \\
\\
30 \\
32 \\
17 \\
23 \\
21 \\
10\end{array}$ & $\begin{array}{l}100 \\
100 \\
\\
100 \\
200 \\
200 \\
165 \\
165 \\
\end{array}$ \\
\hline 12 & $\begin{array}{l}\text { R.S.S. } \\
3110\end{array}$ & 11 & $\mathbf{F}$ & $\begin{array}{l}5 \mathrm{Jt} P \\
7 \mathrm{H} \\
8 \mathrm{Jt} \\
9 \mathrm{C}\end{array}$ & $\begin{array}{l}\text { May 5,1938 } \\
\text { May } 14,1938\end{array}$ & $\begin{array}{l}\text { None. } \\
\text { Mild carditis. }\end{array}$ & few & 9 & $\begin{array}{l}\text { April } \\
\text { May } \\
\text { May } \\
\text { May } \\
\text { May } \\
\text { May } \\
\text { May } \\
\text { May } \\
\text { June } \\
\text { June } \\
\text { June } \\
\text { June } \\
\text { June } \\
\text { July }\end{array}$ & $\begin{array}{r}28 \\
5 \\
13 \\
15 \\
16 \\
22 \\
29 \\
31 \\
4 \\
6 \\
9 \\
12 \\
18 \\
2\end{array}$ & $\begin{array}{r}8,500 \\
8,900 \\
13,900 \\
\\
12,800 \\
8,800 \\
9,500 \\
11,500 \\
11,300 \\
7,900\end{array}$ & $\begin{array}{r}30 \\
31 \\
24 \\
28 \\
\\
21 \\
18 \\
1\end{array}$ & $\begin{array}{r}60 \\
100 \\
165 \\
200 \\
\\
\\
165 \\
165 \\
100\end{array}$ \\
\hline
\end{tabular}


pharynx in most of the cases looked definitely inflamed and the white blood count was elevated with counts ranging from 13,000 to 24,000 in a large proportion of the cases. There were 4 cases in February. The outbreak reached its peak in March with 17 cases and declined gradually with 6 cases in April and 5 in May. Eleven of the 32 children showed a definite rise in antistreptolysin $O$ titer. Fourteen children became carriers of streptococcus Type 4.

Aside from the streptococcus Type 4 infections during March, 6 children developed upper respiratory infections with streptococci other than Type 4. The cultural characteristics of the organisms isolated from these 6 cases were so similar that it was thought possible that they might belong to one serological type. However, it was impossible to identify these strains with any of the known types of streptococci. Attempts to produce agglutinating and precipitating sera by immunizing rabbits with one of the cultures isolated from this group of patients were unsuccessful. Although the serological identity of these 6 strains was not proved, they were tentatively considered to represent the same type, designated as 97T.

The symptoms produced in these 6 children were similar to those associated with the streptococcus Type 4 infections; and these two infections could not be distinguished clinically. Three of these 6 children showed a rise in antistreptolysin O titer. One child contracted both the Type 4 and the $97 \mathrm{~T}$ infections.

None of the 38 children who had these streptococcal upper respiratory infections ( 32 cases due to Type 4, 6 cases probably due to the group of organisms represented by $97 \mathrm{~T}$ ) developed rheumatic sequelae. Nor did the remaining 70 children who escaped streptococcal upper respiratory infections develop clinical or laboratory signs of rheumatic activity.

\section{Streptococcal upper respiratory infections occurring during the winter of 1939 to 1940}

Beginning in November 1939 and continuing until May 1940, 39 of the 108 children developed upper respiratory infections associated with Group A beta hemolytic streptococci of a single type, Type 27. Most of these infections were slightly less severe than those associated with streptococcus Type 4 and streptococcus $97 \mathrm{~T}$ observed during the spring of 1939, but definitely more severe than those associated with streptococcus C51 occurring during the winter of 1937 to 1938 . Following a latent period of twelve to twenty-two days, 8 of 39 children who had upper respiratory infections associated with streptococcus Type 27 developed rheumatic recurrences. The seasonal incidence of these infections and distribution of recurrences are presented in Table II.

TABLE II

The seasonal distribution of streptococcus Type 27 upper respiratory infections and incidence of rheumatic recurrences

\begin{tabular}{|c|c|c|}
\hline $\begin{array}{c}\text { Seasonal } \\
\text { distribution }\end{array}$ & $\begin{array}{l}\text { Number } \\
\text { of cases }\end{array}$ & $\begin{array}{l}\text { Rheumatic } \\
\text { recurrences }\end{array}$ \\
\hline \multicolumn{2}{|c|}{ 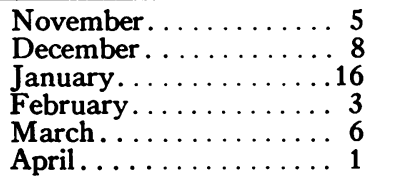 } & $\begin{array}{l}1 \\
2 \\
2 \\
0 \\
2 \\
1\end{array}$ \\
\hline Total. & .39 & 8 \\
\hline
\end{tabular}

The data regarding the 8 children who developed rheumatic recurrences are presented in Table III.

The severity of the pharyngitis varied greatly. Two children had no fever and one had slight malaise with a maximum temperature of $100.4^{\circ} \mathrm{F}$. on one day. The other 5 had definite febrile reactions accompanied by elevated leukocyte counts. With one exception, the number of streptococci present in the throat was large. After a latent period varying from eleven to twenty-two days following the streptococcal pharyngitis, these 8 patients developed clear-cut rheumatic manifestations. Four of the 8 children showed a rise in antistreptolysin $\mathrm{O}$ titer within a month or less following the upper respiratory infection. In 3, a slight or questionable rise occurred after five weeks and in one there was no rise.

Of the 31 children in whom the streptococcal pharyngitis was not followed by rheumatic sequelae, 6 showed a definite rise in antistreptolysin $O$ titer. Three of these 6 children developed cervical adenitis accompanied by a secondary rise in temperature. The remaining 25 children, who escaped rheumatic recurrences, showed no significant rise in antistreptolysin $\mathrm{O}$ titer. 
TABLE III

Data on streptococcal upper respiratory infections with streptococcus $T y p e 27$ followed by rheumatic sequelae in 8 children during the winter 1939 to 1940

\begin{tabular}{|c|c|c|c|c|c|c|c|c|c|c|c|}
\hline \multirow[b]{2}{*}{$\begin{array}{c}\text { Case } \\
\text { num- } \\
\text { ber }\end{array}$} & \multicolumn{3}{|c|}{ Patient } & \multirow[b]{2}{*}{ Date } & \multirow[b]{2}{*}{ Clinical findings } & \multirow{2}{*}{$\begin{array}{l}\text { Strepto- } \\
\text { coccus } \\
\text { Type } \\
27 . \\
\text { num- } \\
\text { ber of } \\
\text { colonies }\end{array}$} & \multirow{2}{*}{$\begin{array}{l}\text { La- } \\
\text { tent } \\
\text { pe- } \\
\text { riod }\end{array}$} & \multicolumn{4}{|c|}{ Blood } \\
\hline & Name & Age & Sex & & & & & Date & $\begin{array}{l}\text { White } \\
\text { blood } \\
\text { cells }\end{array}$ & $\begin{array}{c}\text { Sedi- } \\
\text { ment- } \\
\text { ation } \\
\text { rate }\end{array}$ & $\begin{array}{l}\text { Anti- } \\
\text { strepto- } \\
\text { lygin 0 } \\
\text { titer }\end{array}$ \\
\hline 1 & $\underset{3513}{\text { I.M. }}$ & $\begin{array}{c}\text { years } \\
10\end{array}$ & F & $\begin{array}{l}\text { November 17, } 1939 \\
\text { December 6, } 1939\end{array}$ & $\begin{array}{l}\text { Pharyngitis. Fever } 103.6 \text { to } \\
101^{\circ} \text { for three days. } \\
\text { Polyarthritis, carditis (PR } \\
\text { interval prolonged). }\end{array}$ & $75 \%$ & 19 & $\begin{array}{lr}\text { October } & 17 \\
\text { November } & 6 \\
& \\
\text { November } & 17 \\
\text { November } & 20 \\
\text { November } & 28 \\
\text { December } & 5 \\
& \\
\text { December } & 7 \\
\text { December } & 15 \\
\text { December } & 22 \\
\text { January } & 6 \\
\text { January } & 11 \\
\text { January } & 14 \\
\text { February } & 1 \\
\text { February } & 10 \\
\text { February } & 17\end{array}$ & $\begin{array}{r}8,300 \\
15,200 \\
9,200 \\
\\
13,700 \\
14,800 \\
6,500 \\
8,300\end{array}$ & $\begin{array}{c}m m . \\
1 \\
7 \\
\\
30 \\
31 \\
29 \\
\\
28 \\
23 \\
14 \\
15 \\
12 \\
6\end{array}$ & $\begin{array}{l}\text { units } \\
165 \\
165 \\
\\
100 \\
250 \\
250 \\
\\
250 \\
250 \\
250 \\
200 \\
200\end{array}$ \\
\hline 2 & $\underset{34.57}{\text { H.W. }}$ & 9 & $\mathbf{F}$ & $\begin{array}{l}\text { December 17, } 1939 \\
\text { December 28, } 1939\end{array}$ & $\begin{array}{l}\text { Malaise. Fever } 100.4^{\circ} \text { for } \\
\text { one day. } \\
\text { Carditis. }\end{array}$ & 30 & 11 & 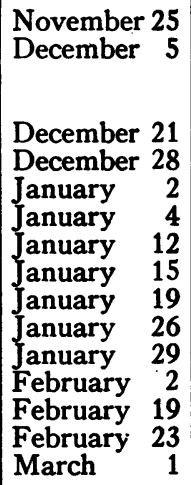 & $\begin{array}{r}5,400 \\
17,000 \\
15,700 \\
11,200 \\
10,500 \\
8,900 \\
7,000\end{array}$ & $\begin{array}{r}5 \\
\\
19 \\
15 \\
\\
32 \\
33 \\
\\
35 \\
28 \\
\\
27 \\
\\
23 \\
16\end{array}$ & $\begin{array}{l}100 \\
100 \\
\\
165 \\
200 \\
250 \\
200 \\
200 \\
200\end{array}$ \\
\hline 3 & O.G. & 13 & $\mathbf{F}$ & $\begin{array}{l}\text { December 22, } 1939 \\
\text { January } 8,1940\end{array}$ & $\begin{array}{l}\text { Pharyngitis, slight adenitis. } \\
\text { Fever } 103 \text { to } 100.2^{\circ} \text { for } \\
\text { three days. } \\
\text { Polyarthritis, carditis (PR } \\
\text { interval prolonged). }\end{array}$ & $80 \%$ & 17 & \begin{tabular}{|rr} 
December & 6 \\
December & 13 \\
& \\
December & 23 \\
December & 26 \\
December & 28 \\
January & 6 \\
& \\
January & 9 \\
January & 15 \\
January & 22 \\
January & 29 \\
January & 31 \\
February & 12 \\
February & 19 \\
February & 26
\end{tabular} & $\begin{array}{r}7,800 \\
17,900 \\
10,600 \\
12,500 \\
\\
11,500 \\
9,900 \\
10,700 \\
9,200 \\
4,500\end{array}$ & $\begin{array}{r}3 \\
28 \\
\\
6 \\
\\
33 \\
33 \\
34 \\
33 \\
27 \\
21 \\
18\end{array}$ & $\begin{array}{r}80 \\
165 \\
250 \\
250 \\
\\
150 \\
150 \\
100\end{array}$ \\
\hline
\end{tabular}

* This boy's sedimentation rate had been continuously elevated from the date of admission, October 11, 1938, a period of over a year. His rheumatic process, therefore, was never considered entirely quiescent. However, the attack of polyarthritis was typical and followed the streptococcal pharyngitis after the usual latent period. 
TABLE III (continued)

\begin{tabular}{|c|c|c|c|c|c|c|c|c|c|c|c|c|}
\hline \multirow[b]{2}{*}{$\begin{array}{l}\text { Case } \\
\text { num- } \\
\text { ber }\end{array}$} & \multicolumn{3}{|c|}{ Patient } & \multirow[b]{2}{*}{ Date } & \multirow[b]{2}{*}{ Clinical findings } & \multirow{2}{*}{$\begin{array}{l}\text { Strepto- } \\
\text { coccus } \\
\text { Type } \\
27 . \\
\text { num- } \\
\text { ber of } \\
\text { colonies }\end{array}$} & \multirow{2}{*}{$\begin{array}{c}\text { La- } \\
\text { tent } \\
\text { pe- } \\
\text { riod }\end{array}$} & \multicolumn{5}{|c|}{ Blood } \\
\hline & Name & Age & Sex & & & & & Date & & $\begin{array}{l}\text { White } \\
\text { blood } \\
\text { cells }\end{array}$ & $\begin{array}{c}\text { Sedi- } \\
\text { ment- } \\
\text { ation } \\
\text { rate }\end{array}$ & $\begin{array}{l}\text { Anti- } \\
\text { streppto- } \\
\text { lysin } 0 \\
\text { titer }\end{array}$ \\
\hline 4 & $\underset{3323}{J} . C$ & $\begin{array}{c}\text { years } \\
13\end{array}$ & $\mathbf{F}$ & $\begin{array}{l}\text { January } 12,1940 \\
\text { January } 24,1940\end{array}$ & $\begin{array}{l}\text { Pharyngitis. No fever. } \\
\text { Fever, erythema multiforme, } \\
\text { knee painful. }\end{array}$ & 8 & days & $\begin{array}{l}\text { December } \\
\text { January } \\
\text { January } \\
\text { January } \\
\text { January } \\
\text { January } \\
\text { January } \\
\text { February } \\
\text { February } \\
\text { February }\end{array}$ & $\begin{array}{r}26 \\
6 \\
8 \\
17 \\
26 \\
29 \\
31 \\
2 \\
9 \\
13\end{array}$ & $\begin{array}{r}9,200 \\
10,100 \\
10,300 \\
11,400 \\
8,500\end{array}$ & $\begin{array}{r}m m . \\
2 \\
3 \\
\\
1 \\
24 \\
\\
27 \\
1\end{array}$ & $\begin{array}{l}\text { unils } \\
100 \\
100 \\
100 \\
\\
100 \\
100\end{array}$ \\
\hline 5 & $\begin{array}{l}\text { R.T.* } \\
3449\end{array}$ & 11 & $\mathbf{M}$ & $\begin{array}{l}\text { January 22, } 1940 \\
\text { February 9, } 1940\end{array}$ & $\begin{array}{l}\text { Pharyngitis. Fever } 103.6 \text { to } \\
101.4^{\circ} \text { for three days. } \\
\text { Polyarthritis. }\end{array}$ & $75 \%$ & 18 & $\begin{array}{l}\text { December } \\
\text { January } \\
\text { January } \\
\text { January } \\
\text { January } \\
\text { January } \\
\text { January } \\
\text { February } \\
\text { February } \\
\text { February } \\
\text { February } \\
\text { February } \\
\text { February } \\
\text { February } \\
\text { March }\end{array}$ & $\begin{array}{r}11 \\
4 \\
13 \\
23 \\
24 \\
26 \\
31 \\
8 \\
10 \\
15 \\
22 \\
24 \\
27 \\
29 \\
21\end{array}$ & $\begin{array}{r}6,400 \\
18,300 \\
11,100 \\
7,900 \\
\\
9,400 \\
\\
10,900 \\
5,700 \\
6,000\end{array}$ & $\begin{array}{l}27 \\
19 \\
35 \\
24 \\
31 \\
\\
28 \\
32 \\
\\
32 \\
29\end{array}$ & $\begin{array}{l}100 \\
\\
100 \\
100 \\
\\
300 \\
300 \\
\\
300 \\
250\end{array}$ \\
\hline 6 & $\begin{array}{l}\text { A.D. } \\
3436\end{array}$ & 13 & $\bar{F}$ & $\begin{array}{l}\text { March 25, } 1940 \\
\text { April 11, } 1940\end{array}$ & $\begin{array}{l}\text { Pharyngitis. Fever } 102.4 \text { to } \\
100.4^{\circ} \text { for two days. } \\
\text { Carditis (PR interval pro- } \\
\text { longed). }\end{array}$ & $75 \%$ & 17 & $\begin{array}{l}\text { March } \\
\text { March } \\
\text { March } \\
\text { April } \\
\text { April } \\
\text { April } \\
\text { April } \\
\text { April } \\
\text { April } \\
\text { April } \\
\text { May } \\
\text { May } \\
\text { May } \\
\text { May } \\
\text { May } \\
\text { June } \\
\text { June } \\
\text { June }\end{array}$ & \begin{tabular}{r|}
15 \\
20 \\
25 \\
$\cdot 1$ \\
4 \\
12 \\
13 \\
18 \\
20 \\
27 \\
2 \\
4 \\
9 \\
11 \\
18 \\
10 \\
24 \\
30
\end{tabular} & $\begin{array}{r}6,200 \\
12,200 \\
10,300 \\
10,900 \\
14,700 \\
10,000 \\
13,200 \\
10,300 \\
13,100 \\
9,100 \\
5,900 \\
6,100\end{array}$ & $\begin{array}{l}35 \\
20 \\
32 \\
25 \\
\\
31 \\
10 \\
12\end{array}$ & $\begin{array}{r}80 \\
80 \\
80 \\
\\
100 \\
100 \\
60\end{array}$ \\
\hline 7 & A.B. & 11 & $\mathbf{F}$ & $\begin{array}{l}\text { March_26,:1940 } \\
\text { April 17, } 1940\end{array}$ & $\begin{array}{l}\text { Pharyngitis. Fever } 102.2 \text { to } \\
100.6^{\circ} \text { for two days. } \\
\text { Carditis. }\end{array}$ & $70 \%$ & 22 & $\begin{array}{l}\text { March } \\
\text { March } \\
\text { March } \\
\text { March } \\
\text { April } \\
\text { April } \\
\text { April } \\
\text { April } \\
\text { April } \\
\text { April } \\
\text { April } \\
\text { May } \\
\text { May }\end{array}$ & $\begin{array}{r}6 \\
9 \\
15 \\
28 \\
1 \\
4 \\
11 \\
15 \\
18 \\
22 \\
25 \\
2 \\
15\end{array}$ & \begin{tabular}{|r|}
4,400 \\
12,300 \\
9,500 \\
\\
10,300 \\
12,500 \\
13,600 \\
8,900 \\
5,900
\end{tabular} & $\begin{array}{r}1 \\
1 \\
3 \\
13 \\
8 \\
19 \\
14 \\
21 \\
6\end{array}$ & $\begin{array}{l}200 \\
200 \\
250 \\
250\end{array}$ \\
\hline
\end{tabular}


TABLE III (continued)

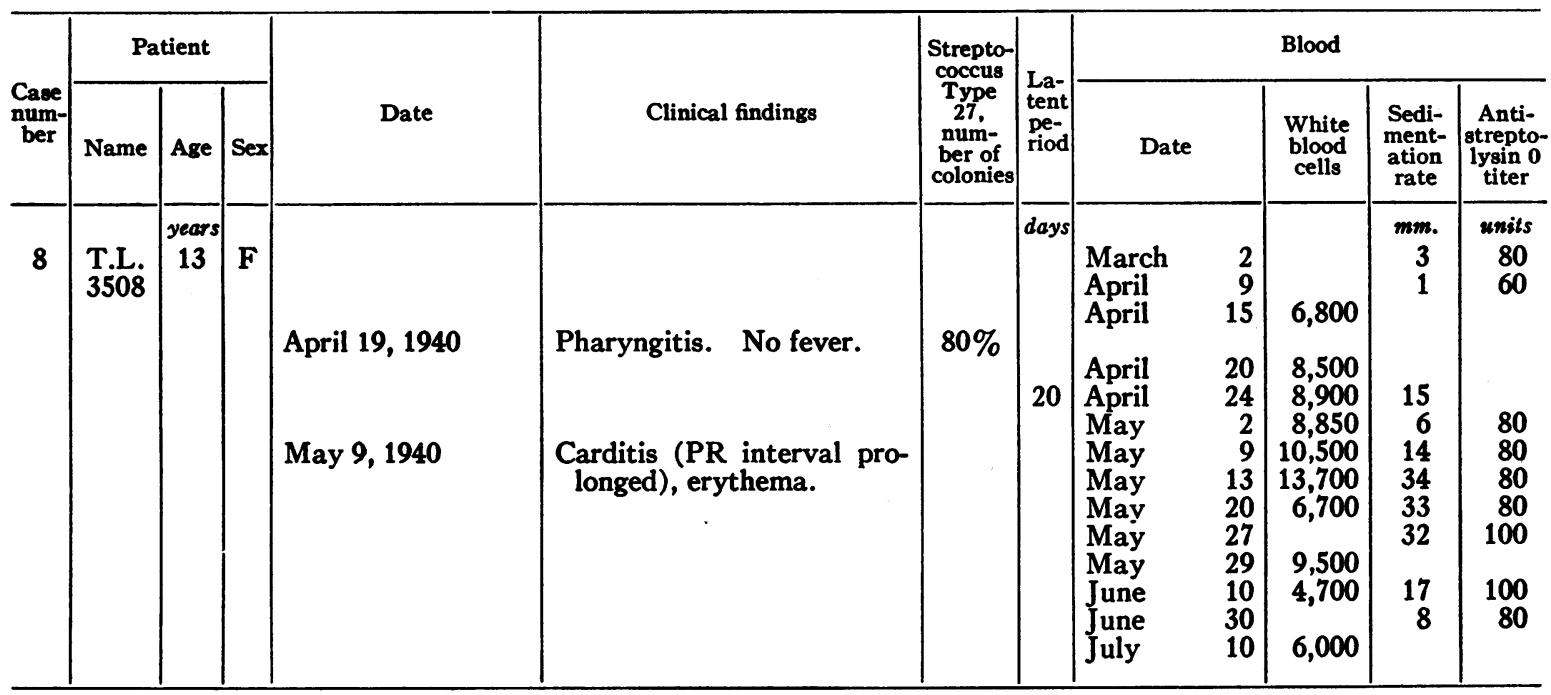

Three children who still had some evidence of rheumatic activity when they were admitted in the fall developed exacerbations during the course of the winter. Twenty-three children who became carriers of streptococcus Type 27 showed no signs of rheumatic activity. None of the remaining 43 children in the institution developed rheumatic manifestations from November 1939 until July 1940.

\section{Comparison of the three epidemic strains isolated 1937 to 1940}

Since the effect of these three outbreaks of streptococcal upper respiratory infections varied greatly in regard to the incidence of rheumatic recurrences, it seemed of interest to compare the three epidemic strains. Therefore, streptococcus C51 (Case 2, Table I), streptococcus Type 4 (Lee) and Type 27 (Case 1, Table III), as well as one of the strains of undetermined type isolated in March 1939, $97 \mathrm{~T}$ (Tudda), were compared as to capsule formation, colony form, mouse virulence, production of the type specific protein " $\mathrm{M}$ " and toxin production.

\section{METHODS}

Capsules. Capsules were studied in India ink preparations with freshly isolated cultures grown from three to five hours in tryptic digest broth. One large loop of culture and one small loop of sterilized India ink were mixed on a plain slide. A coverslip was then pressed down on the drop so as to make a thin layer. The preparation was examined with an oil immersion lens. Only organisms which appeared to be surrounded by a clear area of approximately $1 \mathrm{~mm}$. or more in diameter were considered to have capsules.

Colony type. Broth cultures of recently isolated strains were streaked on freshly prepared 5 per cent rabbit blood agar plates so as to obtain discreet colonies. The plates were sealed with parafilm and incubated for eighteen hours. The watery appearance of typically mucoid colonies was striking. Matt colonies were identified with the aid of a colony microscope.

Mouse virulence. One tenth cc. of an eighteen-hour broth culture of recently isolated strains was injected intraperitoneally into mice, weighing approximately 20 grams. If this amount failed to kill, the culture was considered avirulent. Attempts to raise the virulence of C51, Type 4 and $97 \mathrm{~T}$ by serial mouse passage proved unsuccessful.

Anti " $M$ " precipitating sera. Rabbits were immunized with different strains of the epidemic streptococci C51, Type 4, 97T and Type 27 to determine if precipitins for the type specific protein " $M$ " could be produced. With streptococcus C51 and Type 4, sera were obtained which gave positive precipitin reactions with " $M$ " extracts of the homologous types. To date, the preparation of anti " $M$ " precipitating sera with streptococcus $97 \mathrm{~T}$ and Type 27 has been unsuccessful.

Toxin production. Toxin production was tested by the intravenous injection (7) into rabbits of forty-eight-hour toxin prepared in the usual way (8). For purposes of comparison, a forty-eight-hour toxin was prepared at the same time with the standard toxin strain, NY5. It was found that 1 to $2 \mathrm{cc}$. of the NY5 killed full-grown rabbits regularly in eighteen hours, whereas 10 to $15 \mathrm{cc}$. of the toxins prepared from the other four strains failed to kill. 
The toxin production was further tested on 4 Dickpositive rheumatic children. The NY5 toxin in a dilution of 1:5000 gave positive reactions in all 4 children, whereas the toxins of the four other strains diluted 1:1000 gave irregular results, one child reacting positively and another negatively to the same toxin. In view of the fact that these 4 children were known to have had fairly recent streptococcal upper respiratory infections, it was thought that the positive reactions might be due to sensitivity to streptococcal protein rather than to toxin. These tests, therefore, were considered unreliable.

The results of the comparison of the four strains are presented in Table IV.

TABLE IV

Comparison of the epidemic strains of streptococci

\begin{tabular}{|c|c|c|c|c|c|c|c|c|}
\hline Strain & Year & $\begin{array}{c}\text { Num- } \\
\text { ber } \\
\text { of } \\
\text { cases } \\
\text { U.R.I.* }\end{array}$ & $\begin{array}{l}\text { Num- } \\
\text { ber } \\
\text { of } \\
\text { rheu- } \\
\text { matic } \\
\text { recur- } \\
\text { rences }\end{array}$ & Capsule & Colony & $\begin{array}{c}\text { Mouse } \\
\text { viru- } \\
\text { lence }\end{array}$ & “M”| & $\begin{array}{l}\text { Eryth- } \\
\text { rogenic } \\
\text { toxin }\end{array}$ \\
\hline C51 & $1937-1938$ & 12 & 6 & large & $\begin{array}{c}\text { large } \\
\text { mucoid }\end{array}$ & negative & + & negative \\
\hline $\begin{array}{c}\text { †Type } 4 \\
97 \mathrm{~T}\end{array}$ & $\begin{array}{c}\text { Spring } \\
1939\end{array}$ & $\begin{array}{l}32 \\
6\end{array}$ & $\begin{array}{l}0 \\
0\end{array}$ & $\begin{array}{c}\text { negative } \\
\text { large }\end{array}$ & $\begin{array}{c}\text { medium } \\
\text { matt } \\
\text { mucoíd }\end{array}$ & $\begin{array}{l}\text { negative } \\
\text { negative }\end{array}$ & $\begin{array}{l}+ \\
?\end{array}$ & $\begin{array}{l}\text { negative } \\
\text { negative }\end{array}$ \\
\hline Type 27 & $1939-1940$ & 39 & 8 & large & $\begin{array}{c}\text { large } \\
\text { mucoid }\end{array}$ & negative & ? & negative \\
\hline
\end{tabular}

*U.R.I. = upper respiratory infections.

† Since this paper was accepted for publication, comparative tests on the production of streptolysin 0 and fibrinolysin by the epidemic strains C51, Type 4, and Type 27 have been made. It was found that Type 4 produced a more potent streptolysin 0 and a more active fibrinolysin than either C51 or Type 27.

The comparison of these cultures showed that the only notable difference between the Type 4 organisms, C51, and Type 27 was in the absence of a capsule and in the character of the colony. Although capsulated, mucoid streptococci are usually considered to be more virulent than matt strains, the upper respiratory infections associated with streptococcus Type 4 were more severe than those associated with streptococcus C51 and streptococcus Type 27. It is of interest, furthermore, that during the spring of 1939 when Type 4 was prevalent, 6 children had upper respiratory infections associated with capsulated mucoid streptococci of undetermined types. These 6 children also failed to develop rheumatic recurrences.

Coburn and Pauli (9) have described the isolation of Group A beta hemolytic streptococci which were ineffective in precipitating rheumatic recurrences in apparently susceptible rheumatic indi- viduals. These authors were of the opinion that these strains differed from those associated with the subsequent development of rheumatic manifestations mainly in being poor toxin producers. In the experiments recorded above, no evidence was obtained to indicate that C51 and Type 27 produced particularly strong toxins.

The findings summarized in Table IV suggest that an unknown factor associated with streptococci which precipitates rheumatic fever may have been lacking in Type 4 organisms. Since both C51 and Type 27 appeared capable of precipitating rheumatic recurrences, it can be assumed that these two strains contained or carried the hypothetical special rheumatic factor. The failure of some children to develop rheumatic fever following infections with these two strains might be explained on the basis that these individuals were in a refractory state and therefore were insusceptible to rheumatic fever at that particular time.

It was thought of interest to compare the rheumatic histories of the 31 children who did not develop rheumatic recurrences following the streptococcus Type 27 pharyngitis with those of the 8 who did. Ten of the 39 children, who had upper respiratory infections associated with streptococcus Type 27 during the winter of 1939 to 1940 , had had pharyngitis due to streptococcus Type 4 the previous year. Four children who had rheumatic recurrences following the Type 27 infections had escaped rheumatic sequelae following the Type 4 pharyngitis (Cases 1, 2, 4, and 7, Figure 1).

The rheumatic histories and cardiac findings before and after admission of the 39 children who had the Type 27 pharyngitis are presented in Figure 1.

According to Wilson (2), the rheumatic manifestation of greatest prognostic significance is carditis. Figure 1 shows that a large proportion, 6 out of 8 , of the children who developed rheumatic recurrences following streptococcus Type 27 pharyngitis had had carditis. However, 14 of the 31 children who escaped rheumatic sequelae also had had definite cardiac involvement. Four children who were considered to be particularly vulnerable because their hearts were enlarged failed to develop rheumatic manifestations following the streptococcus Type 27 upper respiratory infection (Cases 23, 29, 35, 37, Figure 1). The average 
age of the 8 children who developed rheumatic recurrences was 11.6 years, and that of the 31 children who escaped was 9.8 years.

Coburn (10) is of the opinion that the type of immune response elicited by streptococcal pharyngitis in rheumatic subjects determines whether or not the rheumatic recurrences will develop. According to this observer, if the rise in antistreptolysin $\mathrm{O}$ titer is delayed rather than prompt, rheumatic sequelae are more likely to occur.

In 9 of the 14 rheumatic recurrences following streptococcal upper respiratory infections observed by us, the rise in antistreptolysin $\mathrm{O}$ titer was not delayed. As already mentioned, 4 children who developed rheumatic manifestations following Type 27 streptococcus pharyngitis had had the non-effective Type 4 streptococcus upper respiratory infection during the spring of 1939 . The immune response of these 4 patients to these two infections was identical. The rise in the antistreptolysin $\mathrm{O}$ titer of 2 of these children, both to the streptococcus Type 4 and Type 27, was marked and prompt. One child failed to show a rise either to the Type 4 or to the Type 27 , and in the other child the rise in both instances was doubtful.
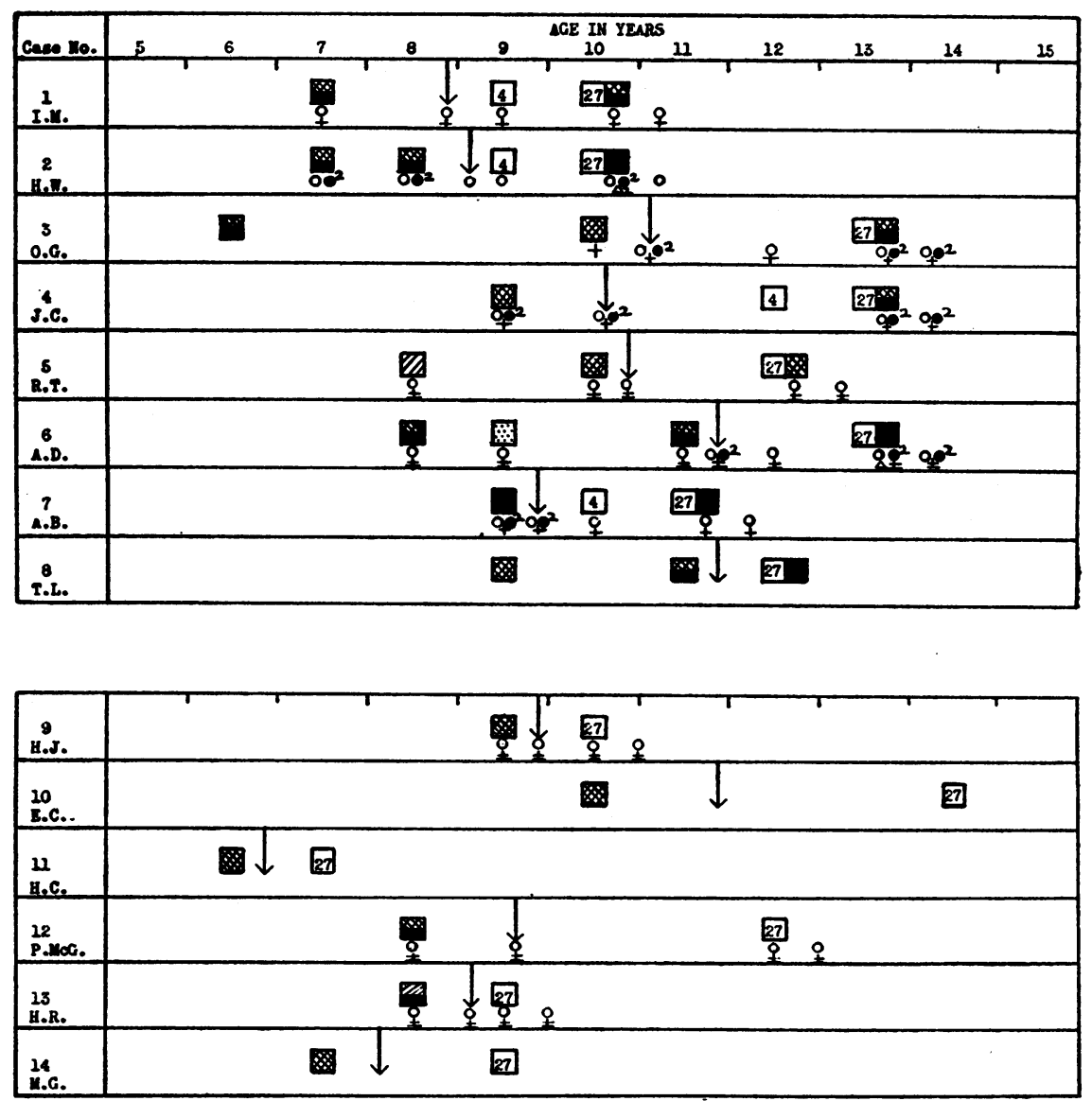

Fig. 1. Rheumatic Histories of 39 Patients Who Developed Streptococcus Type 27 Pharyngitis

Cases 1 to 8 represent the rheumatic histories of the 8 patients who developed rheumatic recurrences. Cases 9 to 14 represent the rheumatic histories of the 6 patients who showed a rise in antistreptolysin $O$ titer without developing rheumatic sequelae. Cases 15 to 39 showed no rise in antistreptolysin $O$ titer and no rheumatic manifestations.

* The symbols used in this Figure were devised by the New York Heart Association. 


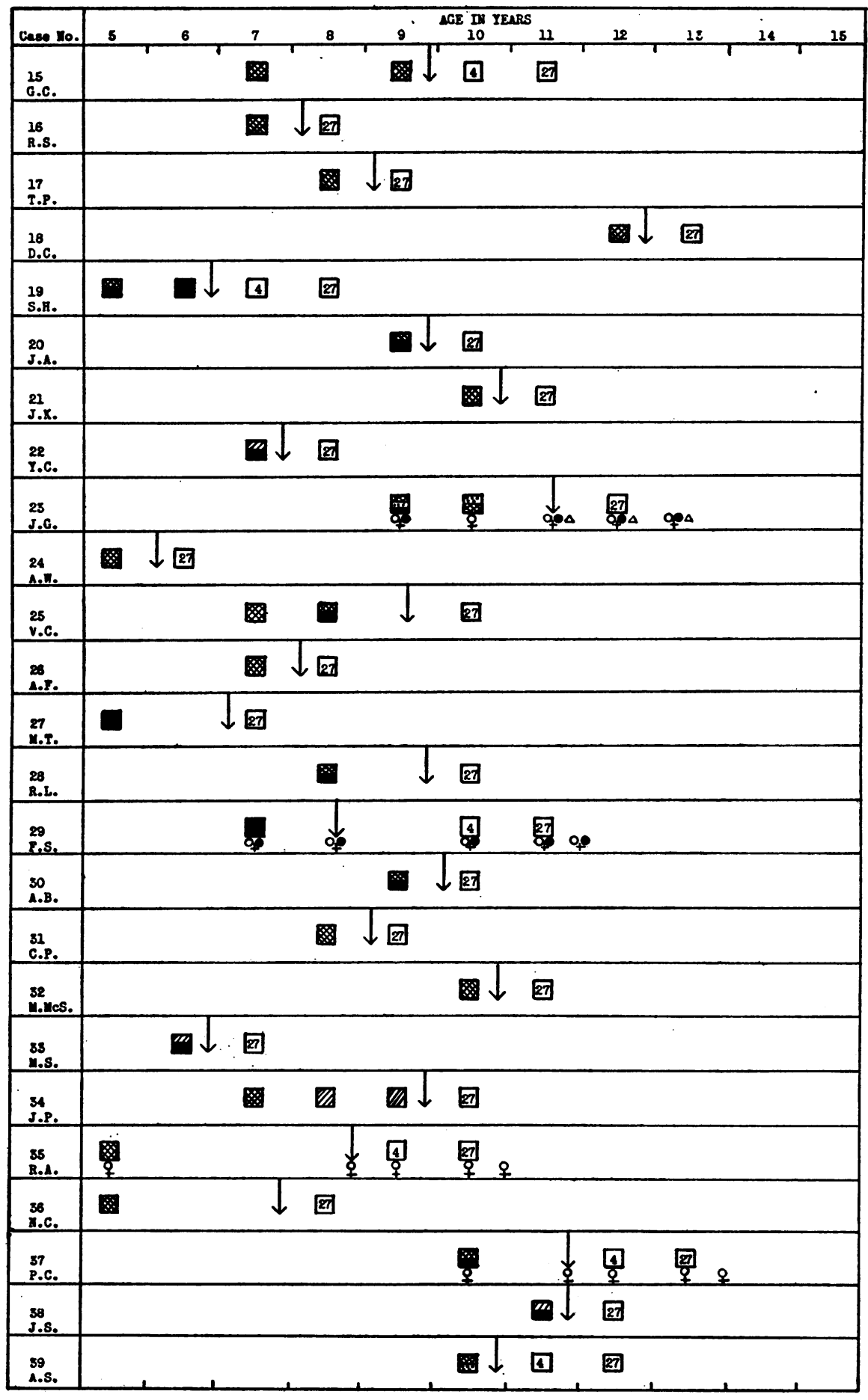

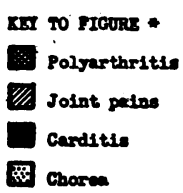

[I]. Stropt. Iypo 4 phargaglts

(7) Strept. Iype 27 pharyagitis

1 Adniseica to Irtington Hiones

- Mitrel Inenrficienery

- Mitral etemosis

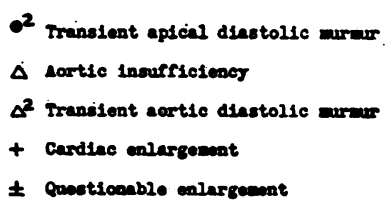

Translent apdeal dingtolic murar

$\Delta$ Aortic ingurficienes

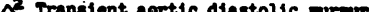

+ Candiac entargenent

Quseticonble calargenent

Frg. 1 
SOURCE OF THE EPIDEMIC STRAINS

In view of the fact that outbreaks of streptococcal upper respiratory infections are considered likely to precipitate rheumatic recurrences, it is the policy of some sanatoria and convalescent homes for rheumatic children to exclude children who are streptococcus carriers. It therefore was thought of interest to trace the probable source of each of the epidemic strains and also report the findings in regard to Group A beta hemolytic streptococcus carriers during each of the three winters.

Children who were found to be streptococcus carriers on admission usually carried small numbers of organisms and therefore were not excluded. Only one boy who had chronic sinusitis with marked purulent nasal discharge, which contained Group A beta hemolytic streptococci in almost pure culture, was sent home immediately.

\section{7 to 1938}

The epidemic strain of this year, streptococcus C51, was introduced by a carrier admitted in May 1937 and discharged in October of that year. During the summer and fall of 1937, 5 other children became carriers of this strain. The first case of streptococcus C51 pharyngitis appeared at the end of December 1937.

Carriers. From December 1, 1937 until June $1,1938,17$ children were carriers of Group A beta hemolytic streptococci other than the epidemic strain C51. No cases of pharyngitis developed which could be attributed to infection with any of these 17 strains.

\section{8 to 1939}

The epidemic strain of this year, streptococcus Type 4, was first isolated from a child with acute pharyngitis. Since streptococcus Type 4 had not been present before, it was thought probable that this child had contracted the upper respiratory infection from her parents who had visited her two days before she became ill. The source of streptococcus $97 \mathrm{~T}$, which was considered the cause of the other 6 cases of pharyngitis occurring during this year, was not determined.

Carriers. From December 1, 1938 until June 1, 1939, 19 children were carriers of Group A beta hemolytic streptococci other than the epidemic strain, streptococcus Type 4. Among these 19 strains, 6 were found to be Types $5,12,17,18$, 19 , and 29 , respectively. One child who had had streptococcus C51 pharyngitis during March 1938 continued to carry this organism until June 14, 1939. The types of the remaining 12 carrier strains were not determined. No cases of pharyngitis developed which could be attributed to infection with any of these 19 strains.

\section{9 to 1940}

The epidemic strain of this year, streptococcus Type 27, was introduced by a child who had a tonsillectomy done in another hospital. She was readmitted two days after the operation. She had fever for five days after her return, and during that time throat cultures showed large numbers of streptococcus Type 27 .

A second strain of undetermined type, designated as R31, was also prevalent during this year. This organism was introduced in June 1939. A large number of children, a total of 22 , gradually became carriers of this strain. Only one child developed a mild pharyngitis due to this organism. After a latent period of fourteen days, this patient showed low-grade symptoms of rheumatic activity.

Carriers. From December 1, 1939 until June 1, 1940, 11 children were carriers of Group A beta hemolytic streptococci other than streptococcus R31 and the epidemic strain, streptococcus Type 27. Four children who were in the institution during the spring of 1939 carried the epidemic strain of that year, streptococcus Type 4, during the winter of 1939 to 1940.

Of the 11 carrier strains 6 were found to be Types $2,4,6,12,17$, and 29 , respectively. The types of the other 5 carrier strains were not determined. No cases of pharyngitis developed which could be attributed to infection with any of these 11 strains.

\section{DISCUSSION}

Three outbreaks of streptococcal upper respiratory infections occurring during three successive winters in a sanatorium for rheumatic children are reported. Each epidemic was associated with Group A beta hemolytic streptococci of a single 
type, but a different type was prevalent in each outbreak. The incidence of rheumatic recurrences following these three waves of streptococcal pharyngitis varied greatly. In the first outbreak, following a latent period, half the children showed signs of rheumatic activity; in the second, all the children escaped rheumatic sequelae; and in the third, a relatively small proportion developed rheumatic recurrences. A comparison of the two types of streptococci associated with recurrences (i.e. " effective" strains) and the " non-effective" strain failed to show any significant differences.

At the present time, there is, in our opinion, no adequate explanation of why rheumatic manifestations sometimes follow in the wake of streptococcal upper respiratory infections and sometimes do not. It seems possible that either the "noneffective" strains of Group A beta hemolytic streptococci lack some unknown, hypothetical factor necessary for the precipitation of rheumatic recurrences, or that the vulnerability of rheumatic subjects to the effect of streptococcal upper respiratory infections varies greatly from year to year.

Following infection with the two "effective" strains of streptococci, rheumatic recurrences developed sporadically over a period of months. It therefore must be assumed that these organisms carried the hypothetical rheumatic factor throughout the epidemics. However, a large number of children who had streptococcal pharyngitis due to these strains failed to develop demonstrable signs of rheumatic activity, suggesting that these individuals may have been in a refractory state. A comparison of the rheumatic histories of the children who did and those who did not develop rheumatic recurrences showed no striking differences.

An analysis of the previous rheumatic attacks of the 14 children who developed rheumatic recurrences following streptococcal upper respiratory infections showed that the rheumatic manifestations in each patient tended to follow a certain pattern characteristic of the particular individual. Mild and severe rheumatic manifestations followed infection with the same strain of Group A beta hemolytic streptococci.

The effect of streptococcal upper respiratory infections in precipitating rheumatic sequelae has usually been studied in rheumatic patients with marked cardiac damage who are known to be sub- ject to frequent rheumatic recurrences. In this study, children in the early stages of rheumatic fever were selected because it is of primary importance to determine what factors precipitate recurrences before marked cardiac damage has developed. It has been found (11) that in the majority of cases severe cardiac involvement occurs within five years after the initial attack of polyarthritis or carditis. At the present time, there is no way of predicting the subsequent course of a child shortly after his first attack of polyarthritis or carditis. It is therefore of interest that 31 children (Cases 9 to 39, Figure 1) who contracted the "effective" streptococcus Type 27 pharyngitis within five years following their first attack of polyarthritis or carditis escaped rheumatic sequelae.

During 1937 to 1938 the probable source of the epidemic strain was a patient who was a streptococcus carrier. During 1938 to 1939 and 1939 to 1940 the probable sources of the epidemic strains were 2 children who developed acute upper respiratory infections as the result of outside contacts. During all three years, a fairly large proportion of the children carried Group A beta hemolytic streptococci of various types which, in most instances, did not spread or cause pharyngitis. It is noteworthy that some of these carrier strains had capsules and formed mucoid colonies. It is our impression that cases of upper respiratory infections due to types of streptococci not previously present in the community are the most likely sources of epidemic strains. Occasionally, a carrier strain may spread slowly from child to child and after several passages produce clinical symptoms of upper respiratory disease. However, at the present time, it is impossible to predict which carrier strain will behave in this way.

In view of these findings, it does not seem to us that the exclusion of rheumatic children from sanatoria or convalescent homes because they are carriers of small or moderate numbers of Group A beta hemolytic streptococci is warranted. One or two throat cultures taken before admission are not adequate to exclude carriers. Children who are carriers may have negative throat cultures for several weeks, or months, and then again show fairly large numbers of streptococci of the same type.

A large proportion of children who have had 
streptococcal pharyngitis continue to carry streptococci for months after recovery. It has been found by several observers (12), as well as ourselves, that although sulphanilamide may reduce the number of streptococci carried temporarily, this drug does not clear up carriers permanently. At the present time, no effective way of clearing up streptococcus carriers is known and it is not feasible to isolate children for months at a time. It therefore is impossible to eliminate Group A beta hemolytic streptococci even in a relatively isolated group of children.

As far as explaining the rôle of Group A beta hemolytic streptococci in the etiology of rheumatic fever is concerned, our observations leave many questions unanswered. Although the total number of rheumatic recurrences, namely fourteen, observed during the three winters among more than 300 rheumatic children is small, it is striking that all of these followed in the wake of streptococcal pharyngitis. No child who escaped streptococcal upper respiratory infections developed rheumatic manifestations. On the other hand, many children who contracted infections with the " effective" strains of streptococci remained well.

At the present time, the situation in regard to rheumatic fever is analogous to that which prevailed in regard to scarlet fever before the discovery of erythrogenic toxin and the Dick test. We now know that toxin-producing strains of streptococci are likely to produce scarlet fever in Dick-positive individuals. In rheumatic fever, we are unable to ascertain the state of susceptibility of the patient or what special property or factor makes certain strains of streptococci " effective." It seems possible that fluctuations in the resistance of rheumatic individuals from year to year may be more important in precipitating rheumatic recurrences than qualitative or quantitative differences between various strains of streptococci.

It is undoubtedly important to continue the study of the relationship of streptococci to the etiology of rheumatic fever. However, another approach to the problem should also be borne in mind. It seems possible that the streptococcus may be merely an indicator which accompanies the invasion or reactivation of a hypothetical rheumatic agent. The entrance or increased activity of this agent may enhance the infectivity of streptococci, just as the multiplication of herpes sim- plex is facilitated by certain diseases which apparently lower the resistance of the patient. If this hypothesis is true, the failure of certain children to develop rheumatic sequelae following pharyngitis due to "effective" strains of streptococci might be explained on the basis that re-infection with exogenous rheumatic "virus" or reactivation of endogenous rheumatic "virus" had not taken place at that particular time. The latent period observed following "effective" streptococcal upper respiratory infections might represent the interval after the new attack or reactivation necessary for the rheumatic agent to multiply sufficiently to produce demonstrable symptoms. Strains of streptococci would then be "effective" or "non-effective" depending on whether or not they were coincident with a fresh infection or a reactivation of the rheumatic agent. The proof of this hypothesis depends on the isolation of a rheumatic "virus."

\section{CONCLUSIONS}

1. The effect of three outbreaks of streptococcal upper respiratory infections during three successive winters in a colony of rheumatic children are described. Each of these outbreaks was due to infections with a single type of Group A beta hemolytic streptococci, but during each epidemic a different type was prevalent.

2. The incidence of rheumatic recurrences following these streptococcal upper respiratory infections varied greatly-from none to a large proportion of the cases.

3. A comparison of the epidemic strains failed to reveal any significant differences which might account for the variations in the incidence of rheumatic recurrences.

4. A comparison of the rheumatic histories of children who escaped and of those who developed rheumatic recurrences following pharyngitis due to " effective" strains of streptococci likewise did not show any striking differences. Our findings suggest that the vulnerability of the rheumatic subject to the effect of streptococcal upper respiratory infections is variable, and depends on factors which at the present time are not understood.

5. No rheumatic recurrences were observed in children who escaped streptococcal upper respiratory infections during the three-year period. 


\section{BIBLIOGRAPHY}

1a. Glover, J. A., Incidence of acute rheumatism. Lancet, 1930, 1, 499.

b. Schlesinger, B., The relationship of throat infection to acute rheumatism in children. Arch. Dis. Childhood, 1930, 5, 411.

c. Glover, J. A., and Griffith, F., Acute tonsillitis and some of its sequels: epidemiological and bacteriological observations. Brit. M. J., 1931, 2, 521.

d. Collis, W. R. F., Acute rheumatism and hemolytic streptococci. Lancet, 1931, 1, 1341.

e. Sheldon, W., On acute rheumatism following tonsillitis. Lancet, 1931, 1, 1337.

f. Bradley, W. H., Epidemic acute rheumatism in a public school. Quarterly J. Med., 1932, 1, 79.

g. Coburn, A. F., and Pauli, R. H., Studies on the relationship of streptococcus hemolyticus to the rheumatic process. III. Observations on the immunological responses of rheumatic subjects to hemolytic streptococcus. J. Exper. Med., 1932, $56,651$.

h. Coburn, A. F., and Pauli, R. H., Studies on the immune response of the rheumatic subject and its relationship to activity of the rheumatic process. II. Observations on an epidemic of influenza followed by hemolytic streptococcus infections in a rheumatic colony. J. Exper. Med., 1935, 62, 137.

i. Coburn, A. F., and Pauli, R. H., III. Observations of the reaction of a rheumatic group to an epidemic infection with hemolytic streptococci of a single type. Ibid., 1935, 62, 159.

2. Wilson, M. G., Rheumatic fever. The Commonwealth Fund, New York, 1940.

3. Krumwiede, E., and Kuttner, A. G., A growth inhibitory substance for the influenza group of organisms in the blood of various animal species. The use of the blood of various animals as a selective medium for the detection of hemolytic streptococci in throat cultures. J. Exper. Med., 1938, 67, 429.

4. Griffith, F., The serological classification of streptococcus pyogenes. J. of Hygiene, 1935, 34, 542.

5. Lancefield, R. C., Antigenic complex of streptococcus haemolyticus; demonstration of type-specific substance in extracts of streptococcus haemolyticus. J. Exper. Med., 1928, 47, 91.

6a. Todd, E. W., Antigenic streptococcal hemolysin. J. Exper. Med., 1932, 55, 267.

b. Todd, E. W., The differentiation of two distinct serological varieties of streptolysin, streptolysin $\mathrm{O}$ and streptolysin S. J. Path. and Bact., 1938, 47, 423.

7a. Hartley, P., Experiments on the purification and concentration of scarlet fever toxin. Brit. J. Exper. Path., 1928, 9, 259.

b. Pulvertaft, R. J. V., On concentration and purification of streptococcal toxin. Brit. J. Exper. Path., 1928, 9, 276.

8. Wadsworth, A., Standard methods of the Division of Laboratories and Research of the New York State Department of Health. Williams and Wilkins, Co., Baltimore, 1927, p. 88.

9. Coburn, A. F., and Pauli, R. H., Studies on the immune response of the rheumatic subject and its relationship to activity of the rheumatic process. IV. Characteristics of strains of hemolytic streptococcus, effective and non-effective, in initiating rheumatic activity. J. Clin. Invest., 1935, 14, 755.

10. Coburn, A. F., Observations on the mechanism of rheumatic fever. Lancet, 1936, 2, 1025.

11. Bland, E. F., and Jones, T. D., Fatal rheumatic fever. Arch. Int. Med., 1938, 61, 161.

12. Rhoads, P. S., and Afremow, M. L., Sulphanilamide in treatment of sore throat due to hemolytic streptococci with controls. J. A. M. A., 1940, 114, 942. 